\title{
Fine Needle Aspiration Cytology (FNAC) in Thyroid Gland Lesions, How Accurate is it? A Correlation with Histopathology Jawher Taher Omer $(\mathrm{PhD})^{1}$ Abstract
}

Background: For the purpose of preoperative diagnosis of thyroid lesions, the Fine Needle Aspiration Cytology (FNAC) has now replaced many other conventional pathology tests. Objective: To know to what extent the FNAC results are accurate by correlating them with results of histopathology.

Patients and Methods: In this retrospective study, ninety-four cases of thyroid gland lesions were collected from the Department of Histopathology at Rizgary Teaching Hospital, Erbil City, Iraq during the period of three years, from January 2015 to December 2017, and correlate their FNAC outcome with that of histopathology.

Results: A total of 94 cases were included in this study, 70 cases (74\%) were female. Fifty cases $(53 \%)$ were between 21-40 years of age. FNAC and histopathological results were correspondent in 84 cases $(89.4 \%)$. The most common thyroid gland lesion was nodular colloid goiter occurred in 72 cases $(77 \%)$ as confirmed by histopathology.

Conclusion: The FNAC is relatively safe, simple, and cost effective preoperative diagnostic tool for thyroid lesions. However, it has some limitations.

Keywords: Thyroid gland lesions, FNAC, Correspondence rate, Histopathology

Corresponding Author: dr.jawher@ymail.com

Received: $19^{\text {th }}$ February 2020

Accepted: $17^{\text {th }}$ May 2020

DOI:https://doi.org/10.26505/DJM.19015270219

\footnotetext{
${ }^{1}$ College of Medicine-Hawler Medical University-Erbil-Iraq
}

\section{Introduction}

Diseases of thyroid gland are of great importance because most are amenable to medical and surgical management, and one of the most challenging tasks a modern physician faces is judging the nature of the thyroid lesion and thereby advocating precise and adequate management of thyroid lesions[1]. Fine-needle aspiration (FNA) for cytology evaluation of thyroid lesions was first reported by Martin and Ellis at New York Memorial Hospital in 1930[2]. The Fine Needle Aspiration Cytology (FNAC) has now become the initial diagnostic procedure that replaced the ordinary pathologic tests for pre-operative diagnosis of thyroid lesions. Currently, the diagnoses of most of benign thyroid lesions are made by the FNAC; this has resulted in an increase in 
Fine Needle Aspiration Cytology (FNAC) in Thyroid Gland Lesions, How Accurate is it? A Correlation

with Histopathology

the incidence of malignancy in thyroidectomy patients from $10 \%$ to $30-50 \%$ in recent years[3]. Although it is the first choice investigation in any thyroid lesion, FNAC has some limitations which are mostly related to sampling techniques, the skill of the physician performing the aspiration, sample adequacy, the experience of cytopathologist in interpreting the aspirate, and overlapping cytological features between benign and malignant follicular neoplasm[4,5]. According to the American Thyroid Association and National Comprehensive Cancer Network guidelines, FNAC should be used as an initial diagnostic test because of its superior diagnostic reliability and cost effectiveness before both thyroid scintigraphy and ultrasonography[6].

The Aim of the study is to evaluate the results of thyroid FNAC and correlate them with that of Histopathology.

\section{Patients and Methods}

In this retrospective study 94 cases of thyroid gland diseases were collected from the Histopathology Department of Rizgary hospital in Erbil City, Iraq during the period from January 2015 to December 2017 who had thyroid gland lesions for whom FNA was performed under ultrasound guidance by trained personnel. The wet smears were prepared by fixing in $95 \%$ ethanol, the slides were stained with Hematoxylin and Eosin (H\&E) stain, and cytological diagnosis was done by specialized cytopathologists. Thereafter, surgically excised thyroid biopsies from the same patients have been fixed in $10 \%$ formalin, examined grossly, processed, and slides were stained with $H \& E$ stain and finally reported by senior histopathologists. A comparison between the cytological and histopathological results was carried out using many clinicopathological parameters to know to what extent the FNA cytological results were accurate by finding out the correspondence rate between both the FNAC results and histopathology report results. We used tables and figures to describe this correlation in addition to gender and age range prevalence, and the predominant thyroid lesion(s) in Erbil City.

\section{Statistical analysis}

Statistical analysis was performed using Microsoft Excel, Office 2010. Data was described in number and percentage. Quantitative data were described using mean, standard deviation median, and range.

\section{Results}

A total of 94 cases were included in this study. Out of 94 cases: 24 cases (26\%) were Male, and 70 cases (74\%) were female as shown

in

Figure 


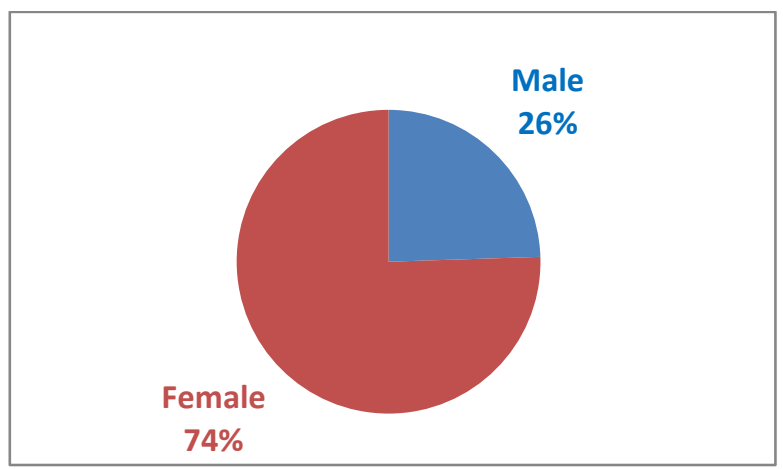

Figure (1): Gender distribution of cases

Table (1) shows the age distribution of the studied cases. Majority [88 cases (94\%)] were between 21 to 60 years.

Table (1): Age distribution

\begin{tabular}{|c||c||}
\hline Age range (in years) & Number of cases $(\%)$ \\
\hline \hline$<20$ & $2(2 \%)$ \\
\hline \hline $21-40$ & $50(53 \%)$ \\
\hline \hline $41-60$ & $38(41 \%)$ \\
\hline \hline$>60$ & $4(4 \%)$ \\
\hline \hline Total & $94(100 \%)$ \\
\hline
\end{tabular}

Table (2) shows the corresponding and non- Histopathology for each thyroid gland lesion corresponding results of FNA-cytology with separately:

Table (2): Level of correspondence

\begin{tabular}{|c|c|c|c|}
\hline \multirow{2}{*}{$\begin{array}{l}\text { Thyroid } \\
\text { diseases }\end{array}$} & \multirow[t]{2}{*}{ No. of cases } & Corresponding & Not corresponding \\
\hline & & No. (\%) & No. (\%) \\
\hline $\begin{array}{l}\text { Nodular colloid } \\
\text { goiter }\end{array}$ & 72 & $72(100 \%)$ & $0(0 \%)$ \\
\hline $\begin{array}{l}\text { Papillary } \\
\text { carcinoma }\end{array}$ & 12 & $8(66.7 \%)$ & $4(33.3 \%)$ \\
\hline $\begin{array}{l}\text { Follicular } \\
\text { adenoma }\end{array}$ & 4 & $0(0 \%)$ & $4(100 \%)$ \\
\hline Thyroid cyst & 2 & $2(100 \%)$ & $0(0 \%)$ \\
\hline $\begin{array}{c}\text { Hashimoto's } \\
\text { thyroiditis }\end{array}$ & 3 & $2(66.7 \%)$ & 10 \\
\hline Graves' disease & $\overline{11}$ & $0(0 \%)$ & $1(100 \%)$ \\
\hline Total & 94 & $84(89.4 \%)$ & $10(10.6 \%)$ \\
\hline
\end{tabular}


Among the 94 studied cases of thyroid gland disorders, the results of cytological examination in 84 cases $(89.4 \%)$ were corresponding to the final histopathology result of the biopsies; while the results were not correspondent in 10 cases $(10.6 \%)$ as shown in Figure

(2):

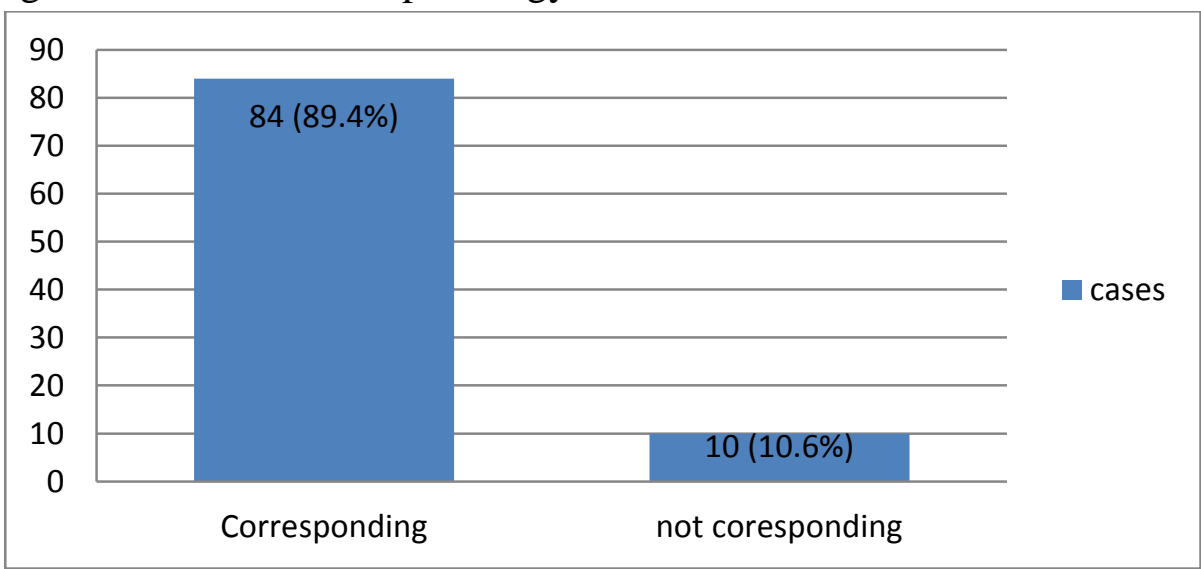

Figure (2): Overall correspondence rate

The final diagnosis of 94 studied cases of histopathological biopsy reports was as thyroid gland lesions depending on the follows: see Figure(3)

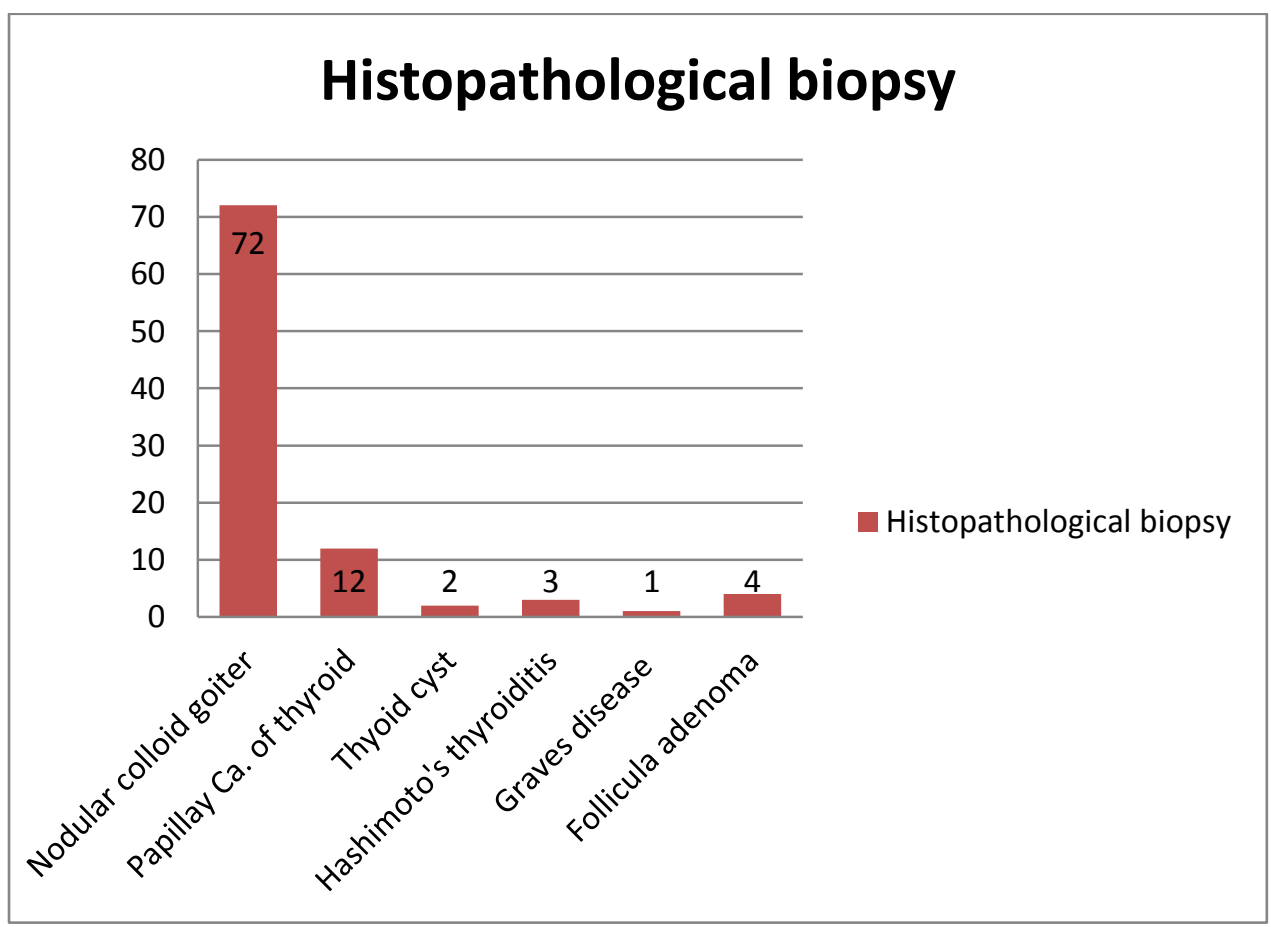

Figure (3): Final Histopatholoical Diagnosis (No. of cases) 


\section{Discussion}

There is a worldwide consensus now to perform FNA of thyroid gland lesions as an initial diagnostic step because it has proven to be more economical and reliable procedure for the identification of thyroid tumors that require surgical excision and others that can be conservatively treated[7].

In this study all FNACs were performed under ultrasound guide for patients with thyroid lesions who were admitted at Rizgary hospital. Both FNAC and histopathology work have been done at the same lab section of the hospital. In the current study it was found that the majority of patients were of female, 70 cases $(74 \%)$; while 24 (26\%) were male Figure (1). Similar results were found in other published studies done by Hamdani and Reshi[8]; Khageswar Rout et al[9]; and Sheela Chaudhari et al[10].

Regarding age distribution, our cases were mostly in third and fourth decade of life, 50 cases $(53 \%)$ as shown in table1. This is in accordance with the studies by Sheela Chaudhari et al.[10], Manoj Gupta et al.[11], Uma Handa et al [12].

The correspondence rate between FNAC and Histopathology report results was $100 \%$ in nodular colloid goiter (72 cases) and thyroid cyst (2 cases); while in papillary carcinoma and Hashimoto's thyroiditis the correspondence rate was 8 out 12 and 2 out of 3 respectively. No correspondence or matching was found between FNAC and histopathology results in both follicular adenoma (4 cases) and Grave's disease (1 case) Table (2). The over all correspondence rate between FNAC and histopathology biopsy findings was $89.4 \%$ (84 cases); while there was disagreement between the two results in 10 cases $(10.6 \%)$ Figure (2). The disagreement between the two diagnostic methods in some diseases like follicular adenoma may be because the differentiation between follicular adenoma and follicular carcinoma of thyroid gland requires the assessment of capsular and vascular invasion by the tumor (absent in adenoma but present in carcinoma) which only seen in tissue sections of excisional biopsy and cannot be recognized in FNAC smears Figure $(7,8)$; while in other conditions like Hashimoto's thyroiditis or Grave's disease it may be due to technical errors during fine needle aspiration process, inadequate aspiration, poor processing, or wrong interpretation of cytological smears and so the microscope features like presence of Hurthle cells in cases of Hashimoto's thyroiditis were not observed in cytology smears; so they just diagnosed as lymphocytic thyroiditis Figure (4). The diagnostic accuracy of FNAC in our study was $89.4 \%$, and this is close to FNAC accuracy results that found in previous studies performed by Hamdani and Reshi[8] (88\%); Manoj Gupta et al.[11] (88.8\%); and Reetika Sharma, et al.[13](90\%). While the accuracy results of FNAC was somewhat differ and with higher records in studies done by: Khageswar Rout et al.[9] (96.05\%); Sheela Chaudhari et al.[10] (94\%); Bagga and Mahajan[14] (96.2\%); and Uma Handa et al.[12] (98.48\%). The most prevalent 
thyroid disease in our study depending on final histopathological diagnosis was nodular colloid goiter, 72 cases $(77 \%)$ followed by papillary carcinoma of thyroid gland, 12 cases (13\%). The least common thyroid lesion was grave's disease, 1 case $(1 \%)$
Figure $(3,5,6)$. Nodular colloid goiter was also the most prevalent thyroid lesion in other studies performed by Sheela Chaudhari et al[10], Bagga and Mahajan[14], and Uma Handa et al[12].

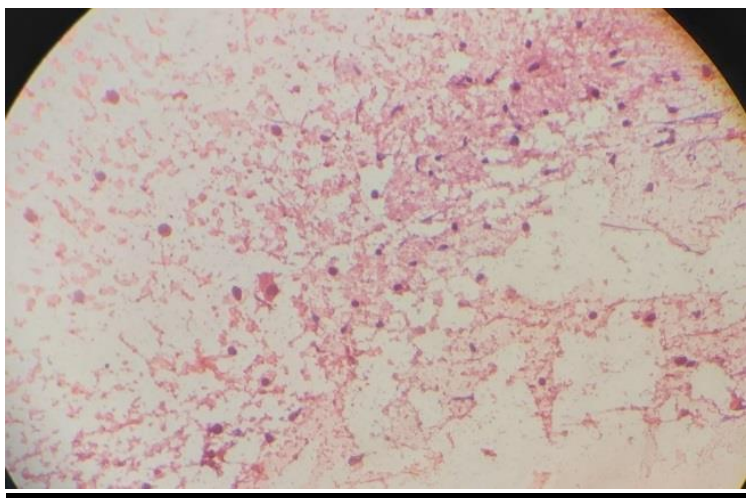

Figure (4): Hashimoto's thyroiditis - cytology slide (H\&E stain x400)

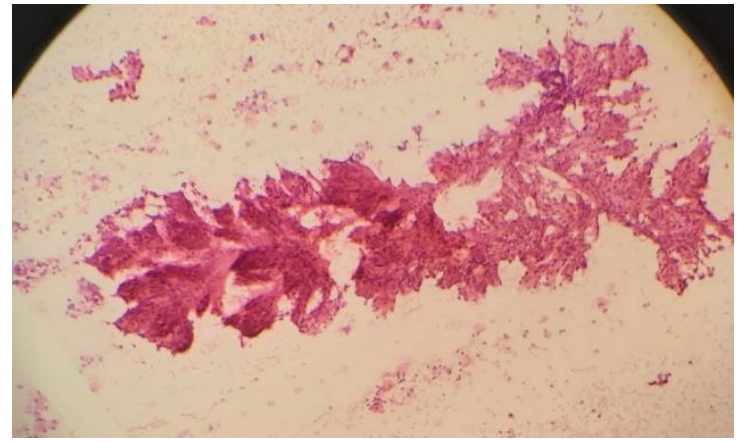

Figure (5): Papillary carcinoma of thyroid gland- cytology slide (H\&EX200)

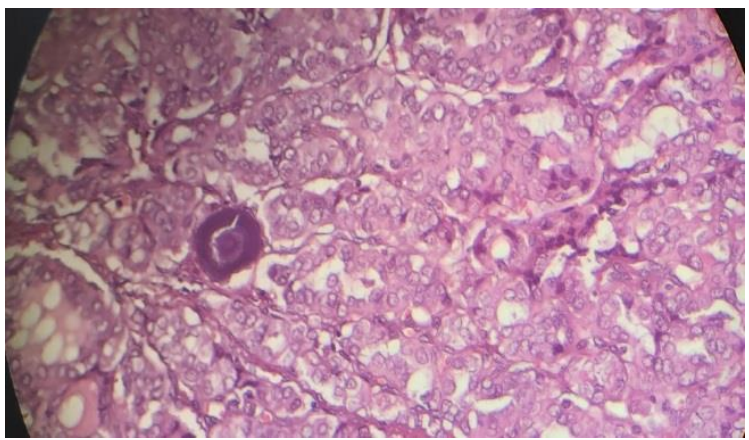

Figure (6): Papillary carcinoma of thyroid gland-histopathology slide(H\&E stainx400) 


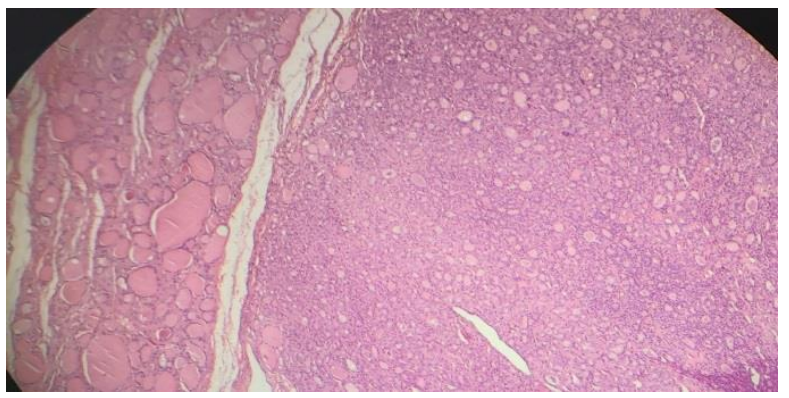

Figure (7): Follicular adenoma of thyroid gland- histopathology slide (H\&E stainx200)

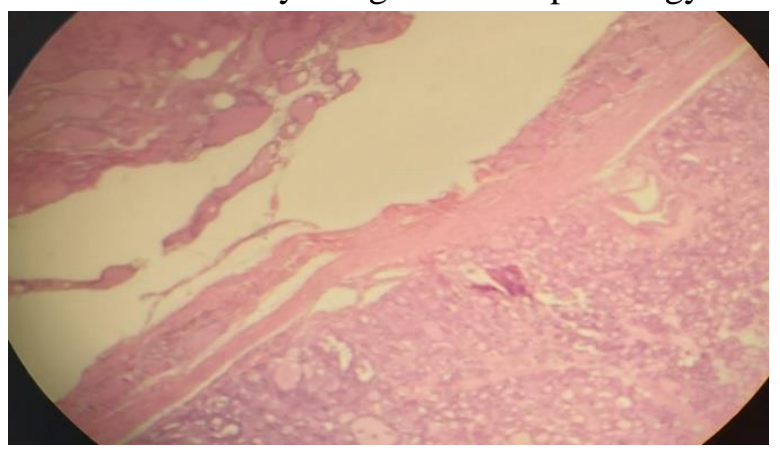

Figure (8): Follicular adenoma of thyroid gland- histopathology slide (H\&E stainx400)

[2]Martin HE, Ellis EB. Biopsy by needle

\section{Conclusions}

We can conclude that FNAC is relatively safe, simple, and cost effective preoperative diagnostic tool for thyroid lesions. However, it has some limitations mostly related to sampling techniques, aspiration, sample adequacy, experience of pathologist, and overlapping cytological features between benign and malignant follicular neoplasms.

\section{Recommendations}

We recommend future works carried out in this field to use more samples and multicenter studies to confirm our results.

\section{References}

[1]Polyzos SA, Kita M, Avramidis A. Thyroid nodules-stepwise diagnosis and management. Hormones

(Athens) puncture and aspiration.

Ann Surg. 1930;92:169-81.

[3]Ridgway EC. Clinical review 30: Clinician's evaluation of a solitary thyroid nodule. J Clin Endocrinol Metab 1992;74:231-5.

[4]Pandey P, Dixit A, Mahajan NC. Fineneedle aspiration of the thyroid: A cytohistologic correlation with critical evaluation of discordant cases. Thyroid Res Pract 2012;9:32-9.

[5]Caraway NP, Sneige N, Samaan NA. Diagnostic pitfalls in thyroid fineneedle aspiration: A review of 394 cases. Diagn Cytopathol 1993;9:345-50.

[6]H.Thyroid: Fine Needle Aspiration (FNA) and cytology. Thyroid 2003;13:80-6. 
Fine Needle Aspiration Cytology (FNAC) in Thyroid Gland Lesions, How Accurate is it? A Correlation

with Histopathology

[7]Nguyen GK, Lee MW, Ginsberg J, Wragg

T, Bilodeau D. Fine-needle aspiration of the thyroid: an overview. Cytojournal 2005;2:12.

[8]Hamdani and Reshi: Correlation of cytodiagnosis with histopathology in thyroid lesions. International Journal of Medical Science and Public Health | 2015 | Vol 4 | Issue 8, P: 1080-1085.

[9]Khageswar Rout ,Chinmay Sunder Ray,Subrat Kumar Behera,and Rudranarayan Biswal. Indian J Otolaryngol Head Neck Surg (October-December 2011) 63(4):370372.

[10]Sheela Chaudhari, Deepa Hatwal, Pawan Bhat, Neha Batra, and Sachan Bhat. International Journal of Scientific Study | November 2015 | Vol 3 | Issue 8; P: 132-135. [11]Manoj Gupta, Savita Gupta, and Ved Bhushan Gupta. Journal of Thyroid Research Volume 2010, Article ID 379051, 5 pages doi:10.4061/2010/379051.

[12]Uma Handa, Sukant Garg, Harsh Mohan, Nitin Nagarkar; Journal of Cytology / January 2008 / Volume 25 / Issue 1.

[13]Reetika Sharma, et al.: Diagnostic accuracy of FNAC of thyroid gland lesions: Journal of Cancer Research and Therapeutics - Volume 13 - Issue 3 - July-September 2017. [14]Bagga and Mahajan: Fine needle aspiration cytology of thyroid swellings: Indian Journal of Cancer | OctoberDecember 2010 | Volume 47 | Issue 4. 